\title{
High resistance to Sacbrood virus disease in Apis cerana (Hymenoptera: Apidae) colonies selected for superior brood viability and hygienic behavior
}

\author{
Nguyen Ngoc VunG ${ }^{1,2}$, Yong Soo $\mathrm{CHOI}^{1}$, $\mathrm{Iksoo} \mathrm{KIM}^{2}$ \\ ${ }^{1}$ Department of Agricultural Biology, National Institute of Agricultural Sciences, Rural Development Administration, \\ Jeonju, Republic of Korea \\ ${ }^{2}$ Department of Applied Biology, College of Agriculture \& Life Sciences, Chonnam National University, Gwangju, \\ Republic of Korea
}

Received 10 March 2019 - Revised 17 July 2019 - Accepted 24 October 2019

\begin{abstract}
Sacbrood virus (SBV) caused significant colony collapse in Korean Apis cerana . Therefore, breeding of resistant bees to counter this viral disease is urgently needed. Considering that hygienic behavior in honey bees confers colony-level resistance against brood diseases, we utilized this trait for selecting A. cerana colonies. In addition, the brood survival rate was evaluated after colonies were SBV-inoculated. Over four selective generations, dead brood removal and brood survivorship in selected colonies were higher than those in the controls, which were not selectively bred for those traits $(P<0.01,99.3 \mathrm{vs} .89 .9 \%$ for removal of pin-killed pupae; $P<0.01,99.0 \mathrm{vs}$. $63.9 \%$ for removal of SBV-killed larvae; and $P<0.01,70.0$ vs. $9.2 \%$ for brood survivorship). Following SBV inoculation, selected colonies showed an increase in the number of surviving pupae and adults, whereas control colonies collapsed mostly. Our results confirm the feasibility of selecting SBV-resistant A. cerana.
\end{abstract}

\section{Apis cerana / bee breeding / Sacbrood virus / instrumental insemination / brood disease}

\section{INTRODUCTION}

Apis cerana Fabricius (Hymenoptera: Apidae) is one of the most important domesticated bee species for the production of high-value bee products and due to its excellent pollination ability, plays an important role in maintaining the biodiversity of plants in Asian countries (Corlett 2004). However, A. cerana populations have greatly decreased in recent years and some populations are near to extinction across Asian countries (Theisen-

Electronic supplementary material The online version of this article (https://doi.org/10.1007/s13592-019-00708-6) contains supplementary material, which is available to authorized users.

Corresponding author: I. Kim,

ikkim81@chonnam.ac.kr

Manuscript editor: Michelle L Flenniken
Jones and Bienefeld 2016). The prime reason for A. cerana colony losses is the Sacbrood virus (SBV) infection. Since its first observation in 2008, SBV has caused more than $90 \%$ of mortality in South Korea (Choi et al. 2010).

The SBV is a linear positive single-stranded RNA virus belonging to the genus Iflavirus in the family Iflaviridae (Baker and Schroeder 2008). The virus primarily affects the brood stage of honey bees, with high viral replication causing significant morphological alterations and ultimately larval death (Bailey et al. 1964) and is transmitted horizontally through SBVcontaminated food as well as vertically through queens and drones (Shan et al. 2017).

As social insects, colony members of honey bees can perform both individual and collective defenses to combat diseases and to promote the 
increase of fitness of the entire colony (Evans et al. 2006; Cremer et al. 2018). Individual defenses typically rely on physiological changes that limit pathogen development into the host (i.e., immune system) and behavioral processes that reduce the risk of pathogen infection (Siva-Jothy et al. 2005; Evans et al. 2006; Brutscher et al. 2015). However, previous studies have shown that genes involved in the innate immunity of honey bees are reduced to a third of those observed in other insects (Honeybee Genome Sequencing Consortium 2006; Evans et al. 2006; McMenamin et al. 2018). Instead, honey bees rely largely on social immunity to defend against diseases (Cremer et al. 2018). Therefore, in honey bees, breeding strategies targeting traits related to both individual and social immunities might be effective for the selection of disease-resistant bees.

Among the social immunity of honey bees, the hygienic behavior, which may exist in all eusocial insects (Cremer and Sixt 2009), plays an important role in the reduction of the loads and transmission rates of pathogens in colonies (Spivak and Reuter 2001; Cremer et al. 2018). The hygienic behavior in honey bees involves the ability of workers to detect and remove diseased, dead, and parasitized broods from colonies (Rath 1999; Lin et al. 2016). Hygienic behavior was reported to be a heritable behavioral trait against brood diseases of honey bees and is commonly considered as a target trait in honey bee breeding programs aiming to improve the vitality of colonies (Gilliam et al. 1983; Milne 1985; Rosenkranz et al. 1993; Spivak and Reuter 2001).

Honey bees are social hymenoptera with the highest levels of multiple mating and as a result, maintain a high level of intracolony genetic diversity (Oldroyd et al. 1998; DeFelice et al. 2015). The genetic diversity of patrilines leads to inheritance of different alleles, including those related to disease resistance, by $F_{1}$ offspring, allowing both colonies and individuals to have various levels of immune responses. Thus, it is less likely for a particular pathogen to cause an entire colonylevel infection (Evans and Spivak 2010). Therefore, for the development of bees that are resistant against diseases, it is important to evaluate individuals' capacities for disease resistance in order to use such traits as breeding sources.
Therefore, in this study, we selected colonies that performed superior hygienic removal of pinkilled pupae and SBV-killed larvae and exhibited higher levels of brood survivorship. According to the results of evaluations on the hygienic behavior and brood viability of colonies in each generation, the best performing colonies (selected from subsets of each line) were used either to rear daughter queens or to produce drones. After four selective generations, the selected $A$. cerana colony showed high resistance against SBV. Thus, this study provides a practical method for the selection of SBV-resistant honey bee lines.

\section{MATERIALS AND METHODS}

\subsection{Bee sources}

A collection of 40 indigenous Korean A. cerana colonies originated from Gumi, Gangjin, Ichon, and Tongyeong, which are located at least $100 \mathrm{~km}$ away from each other and were used for breeder colonies. In each location, 10 colonies were chosen from several apiaries in which the breeders had annually been undergone phenotypic selection for the absence of visible signs of SBV. The selected colonies also exhibited superior apicultural characteristics, such as larger worker population and solid patterns of sealed brood. The colonies were then located in the National Institute of Agricultural Sciences, Jeonju City, Korea $\left(36^{\circ} 49^{\prime} 30^{\prime \prime}\right.$ N, 127 $7^{\circ} 3^{\prime \prime}$ E) in 2015. Colonies originated from Tongyeong served as control for each generation.

\subsection{Breeding and selection procedures}

The main targets for the selection of SBV resistant A. cerana colonies were traits related to superior hygienic behavior and high brood survival rate. The performance of colonies based on those traits was compared with that of control colonies in order to select those that exhibited the highest level of hygienic behavior and brood viability. The best performing colonies $(n=6)$ from each line were selected either to rear daughter queens or to produce drones that contributed semen for artificial insemination of virgin queens. 
The collected colonies were maintained in an apiary for the observation of natural SBV infection from autumn 2015. In early spring 2016, the colonies collected from four locations (four primary groups) displaying better winter survivorship, larger population, and absence of SBV symptoms were subjected to assay for hygienic behavior by pin-killing method (see detailed description of pin-killed assay in "Pin-killed pupal removal test" section). Three most populous colonies from the four primary groups that exhibited over $80 \%$ of dead pupal removal within $24 \mathrm{~h}$ were selected as parent colonies in the first generation. Briefly, we reared queens from the colonies that originated from Gumi (three colonies) and Tongyeong (three colonies) and drones from the colonies that originated from Ichon (three colonies) and Gangjin (three colonies; Figure 1). In the first generation, virgin queens reared from Gumi colonies were instrumentally inseminated with semen of the drones from Ichon colonies to generate line $\mathrm{H}$. Likewise, line $\mathrm{R}$ was the result of crossbreeding between the virgin queens reared from Tongyeong colonies and the drones from Gangjin colonies. Fifteen colonies of each line (i.e., H and R) were generated in April 2016. From the second to the fourth generation, lines $\mathrm{H}$ and $\mathrm{R}$ were maintained separately. In the fourth generation, line $\mathrm{R} \times \mathrm{H}$ was the result of crossbreeding between the virgin queens reared from $\mathrm{R}$ colonies and the drones reared from $\mathrm{H}$ colonies (Figure 1). In each generation, at least 15 colonies for each line were generated.

In each generation, daughter queens were artificially reared from three selected mother colonies of each line (i.e., lines $\mathrm{H}$ and $\mathrm{R}$ ) by grafting 1-dayold worker larvae into cell cups and suspending them vertically on a queen cell frame in a queenless nursery colony. To obtain virgin queens, queen-destined broods were reared in SBV-infected nursery colonies, which had been SBV-inoculated one week prior to queen rearing. The resulting queen-destined broods were developed inside their respective cells in nursery colonies and were transferred to individual queen cages $(3.5 \times 10 \times 1.5 \mathrm{~cm})$ ten days after grafting. Queen cells were then kept in an incubator (Growth chamber, Vision VS-1203PFHLN, Korea) at $34{ }^{\circ} \mathrm{C}$ and $60 \%$ relative humidity until the queen's emergence. Virgin queens were introduced to newly established queenless colonies containing $\sim 4000$ workers in the four brood combs $(39 \times 23 \mathrm{~cm})$ in a modified Langstroth 10 -frame hive box $(41 \times 41 \times 25 \mathrm{~cm})$. The entrance of hives was blocked using a queen excluder (a plastic grid that only allows workers to pass through but restricts the movement of queens) to prevent natural mating of virgin queens.

For each generation, all daughter queens were instrumentally inseminated with a mixture of semen collected from drones raised in three different selected colonies of the same line. Drones raised in different colonies were marked with different colors on the thorax at their emergence to ensure that drones used for instrumental insemination were from the correct breeder colonies. Semen of drones from the same line was collected with $100-\mu \mathrm{L}$ glass capillary of Harbo's syringe (Cobey et al. 2013). The harvested semen was then mixed and homogenized following the protocol described in Cobey et al. (2013). Four microliter of homogenized semen was inseminated to a 6-dayold virgin queen using a Schley insemination apparatus (Schley Model II, Germany) following insemination protocol (Cobey et al. 2013). In each generation, $\sim 22-24$ virgin queens of each line were instrumentally inseminated to have at least 15 egg-laying queens.

\subsection{SBV diagnosis and quantitative real- time PCR}

Larvae with overt symptoms of SBV infection were sampled from SBV-infected A. cerana colonies. We diagnosed SBV disease and six following viruses on Korean A. cerana (Choe et al. 2012), namely, Acute bee paralysis virus (ABPV), Black queen cell virus (BQCV), Chronic bee paralysis virus (CBPV), Deformed wing virus (DWV), Israeli acute paralysis virus (IAPV), and Kashmir bee virus (KBV), using reverse transcription polymerase chain reaction (RT-PCR) protocols described by Choi et al. (2010) and Choe et al. (2012). RNA extraction was performed using RNeasy Mini kit (Qiagen, Germany). Total RNA $(1 \mu \mathrm{g})$ were used for cDNA synthesis using EcoDry premix (Takara, Japan). Primers used for PCR assays were listed in Online resource 1. The 


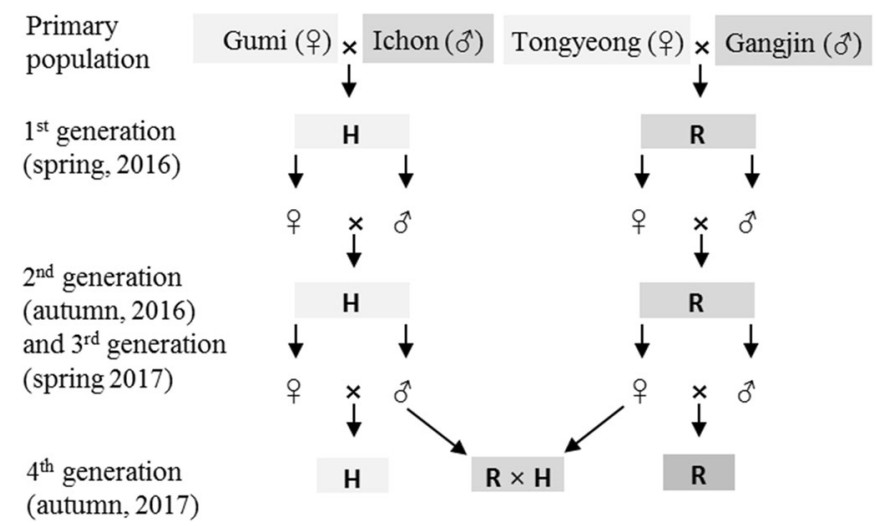

Figure 1. Diagrammatic illustration of breeding procedures. Four generations were propagated from four $A$. cerana populations collected in Gumi, Ichon, Gangjin, and Tongyeong in South Korea. In each generation, three colonies from six selected colonies were used to rear drones, whereas the other three colonies were used to rear daughter queens.

SBV concentration was then quantified using absolute quantification PCR (qPCR) methods described by $\mathrm{Wu}$ et al. (2017). The RT-qPCRs were performed in triplicate in a QuantStudio-5 (Applied Biosystems, USA) using SYBR green (AccuPower 2X GreenStar qPCR MasterMix; Bioneer, Korea). The successful amplification of reference genes ( $\beta$-Actin; Online resource 1 ) was used to confirm the integrity of samples throughout the entire procedure, from RNA extraction to qPCR.

\subsection{SBV inoculation}

We collected larvae with overt symptoms of SBV infection (Gong et al. 2016) from a SBVinfected $A$. cerana colony that had been been confirmed, using RT-PCR assays as described above, for the presence of SBV infection and absence of the infections of the other six viruses described above. The procedure to obtain SBV lysate was conducted as described by Gong et al. 2016. An aliquot of $100-\mu \mathrm{L}$ resultant solution was used for SBV quantification using RT-qPCR assay, as described above. The SBV concentration for inoculation was titrated to $\sim 2 \times 10^{6}$ copies $/ \mu \mathrm{L}$. As SBV is mainly horizontally transmitted through SBV-contaminated food (Chen and Siede 2007) and in order to provoke SBV infections in experimental colonies, both larvae and workers were orally inoculated. Briefly, 500 young larvae (i.e., 1st to 2 nd instar larvae) on a comb of each experimental colony were fed with SBV solution by trickling a dose of $5-\mu \mathrm{L}$ SBV solution to each larva. The workers of each experimental colony were bulk-fed 200-mL sugar syrup (50\% sugar) mixed with $2 \mathrm{~mL}$ of the SBV solution using an internal feeder. These procedures were repeated twice in a $24-\mathrm{h}$ interval. Two weeks after SBV inoculation, larvae with overt symptoms of SBV infection and white-eyed pupae from colonies of each line $(\mathrm{R}, \mathrm{H}$, and $\mathrm{R} \times \mathrm{H})$ and the control in the fourth generation were sampled for SBV quantification using RT-qPCR protocol.

\subsection{Pin-killed pupal removal test}

The ability of workers to remove pin-killed pupae was assayed following the method described by Newton and Ostasiewski (1986). Briefly, a sealed brood comb section containing 100 worker pupae with white eyes $(\sim 8-9$ days after egg hatching) were chosen. Each pupa was killed by piercing the pupal bodies to the bottom of the cell with a needle through the cell capping. The number of empty cells from which killed pupae had been removed by workers was recorded twice, 24 and $48 \mathrm{~h}$ after pin-killing. In each generation, the hygienic test was initiated after queens were allowed to lay eggs for at least 6 weeks; at which time, the workers that were old enough to carry out the hygienic behavior were the offspring 
of the experimental queens. Experiments were triplicated at 3-day intervals.

\subsection{SBV-killed larval removal test}

The purpose of this test was to evaluate the hygienic response of workers against cells containing actual SBV-killed larvae. The selection of individual SBV-killed larvae on a brood comb derived from a SBV-infected colony was inspected visually based on its overt symptoms (Gong et al. 2016). When the SBV-killed larvae were detected on the comb, 100 SBV-killed larvae were indirectly marked. Briefly, we placed a transparent plastic sheet $(39 \times 23 \mathrm{~cm})$ over the surface of the comb and secured it with thumb tacks. Then, we marked this sheet according to its position on the frame of the comb in order to be able to accurately place it back on the comb in the subsequent observations. Finally, we marked the locations of 100 cells containing SBV-killed larvae on this transparent plastic sheet using a permanent marker. When the positions of all cells containing SBV-killed larvae were marked, the transparent plastic sheet was removed and the comb was introduced in the experimental colony. The comb was examined daily for two consecutive days to record if any changes occurred in individual cells. At each observation, the comb was removed from its hive and the transparent plastic sheet was placed on the surface of the comb and aligned with original points of reference to count the cells in which the SBV-killed larvae were completely removed by workers. Thus, the number of SBVkilled larvae removed daily was the result of summing up the empty cells recorded at each observation. For this test, each colony of the breeding lines and control received one comb containing SBV-killed larvae on the same day.

\subsection{Larval survivorship in SBV-infected colonies}

This survival test involved the inoculation of colonies with SBV and the evaluation of the survival rate of larvae at all larval stages, from 1st to 5th instar larvae. Five days after the SBV inoculation, an open brood comb containing newly hatched larvae was taken out from its hive. One hundred 1st instar larvae were indirectly marked on a transparent plastic sheet (as described above in the "SBV-killed larval removal test" section). After the locations of all cells containing 1st instar larvae were marked, the comb was immediately returned to its respective hive. The comb was examined daily for five consecutive days to see if any changes occurred in individual cells. At each observation, abnormal larvae, which exhibited overt symptoms of SBV infection or were partially removed by workers, were all included in the mortality count. Consequently, the number of living larvae at each age was the result of summing the cells containing normal larvae counted daily. This survival test was triplicated at 6-day intervals.

\subsection{Colony strength}

In order to evaluate the impact of SBV on the strength of colonies, colonies of breeding lines and control were SBV-inoculated. The SBV inoculation was conducted after the queens in colonies of breeding lines and control were allowed to lay eggs for 7 weeks in each generation. Following SBV inoculation, the number of pupae and workers in each colony were counted every 15 days for three times using a gridded frame (the size of the bee comb, $39 \times 23 \mathrm{~cm}$ ) consisting of 28 compartments with the size of $4.6 \times 4.6 \mathrm{~cm}$. Briefly, we overlaid the gridded frame on one side of the comb to count all workers in 28 square compartments. Then, we counted the square compartments containing pupae and extrapolated the number of square compartments representing the pupa on one side of the comb to the actual counted number of pupae by multiplying the total square compartments by 100 (number of pupae per square compartment). The same process was repeated for both sides of every comb in each colony. Consequently, the total number of pupae and workers in each colony was the result of summing the individuals in all combs.

\subsection{Data analysis}

Kaplan-Meier estimates followed by log-rank (Mantel-Cox) post hoc tests were used to describe and compare the hygienic abilities (i.e., removal of pin-killed pupae and SBV-killed larvae), larval survivorship, and queen brood survival among 
colonies of breeding lines and the control over time. Data on the number of pupae and workers and area of stored honey were analyzed using repeated measures ANOVA for overall differences of these measured variables among colonies of breeding lines and the control and were analyzed using one-way ANOVA tests followed by Fisher's least significant difference (LSD) post hoc tests for pairwise comparisons of means at individual observation times. All data sets were analyzed using IBM SPSS statistic 22.

\section{RESULTS}

\subsection{Removal of pin-killed pupae}

The removal proportions of pin-killed pupae among breeding lines (lines $\mathrm{R}$ and $\mathrm{H}$ ) in the 1st generation were not significantly different from the control (in the log-rank chi square test; $\chi^{2}=$ $1.47, P=0.48$; Figure $2 \mathrm{a}$ ), but these proportions were significantly different from the control in the 2nd, 3rd, and 4th generations $\left(\chi^{2}=14.01, P<\right.$ 0.01 in the 2 nd generation, Figure $2 b ; \chi^{2}=87.37$, $P<0.01$ in the 3 rd generation, Figure $2 \mathrm{c}$; and $\chi^{2}$ $=128.22, P<0.01$ in the 4 th generation, Figure $2 d$ ). In the 4 th generation, removal proportions of pin-killed pupae in $\mathrm{R}$ and $\mathrm{H}$ colonies increased to $97.9 \%$ and $98.9 \%$ at $48 \mathrm{~h}$, respectively (Figure 2d), indicating a substantial improvement of $8 \%$ and $9 \%$, respectively, when compared with those of the control (Mantel-Cox tests; $P<$ 0.01 in both pairwise comparisons). In particular, fourth-generation colonies of the $\mathrm{R}$ and $\mathrm{H}$ crossline $(\mathrm{R} \times \mathrm{H})$ showed the highest removal proportion of pin-killed pupae $(99.3 \%$ at $48 \mathrm{~h}$, Figure $2 \mathrm{~d}$ ) among the whole experimental group.

\subsection{Removal of SBV-killed larvae}

Removal proportions of SBV-killed larvae among experimental groups in the 1st generation were similar and insignificantly different to the control ( $\chi^{2}=0.28, P=0.87$, Figure 3a). However, these proportions were significantly different from the control at the $2 \mathrm{nd}$, 3rd, and 4th generations $\left(\chi^{2}=9.04, P<0.05\right.$ in the 2 nd generation, Figure $3 \mathrm{~b} ; \chi^{2}=56.03, P<0.01$ in the $3 \mathrm{rd}$ generation, Figure $3 \mathrm{c}$; and $\chi^{2}=451.95, P<$
0.01 in the 4 th generation, Figure $3 d$ ). In the 4 th generation, removal proportions of SBV-killed larvae in $\mathrm{R}$ and $\mathrm{H}$ colonies increased to $95.7 \%$ and $94.3 \%$ at $48 \mathrm{~h}$, respectively (Figure $3 \mathrm{~d}$ ), indicating an increase of $31.8 \%$ and $30.4 \%$, respectively, when compared with those of the control ( $P<0.01$ in both pairwise comparisons). The $\mathrm{R} \times$ $\mathrm{H}$ colonies showed the highest removal proportion of SBV-killed larvae $(99 \%$ within $48 \mathrm{~h}$, Figure $3 \mathrm{~d}$ ) among the whole experimental group.

\subsection{Larval survivorship in SBV-infected colonies}

The proportion of larval survivorship in SBVinfected colonies among experimental groups in the 1 st and 2 nd generations were not significantly different to the control $\left(\chi^{2}=0.67, P=0.7\right.$ in the 1st generation, Figure $4 \mathrm{a}$; and $\chi^{2}=5.88, P=$ 0.053 in the 2 nd generation, Figure $4 b$ ), but these proportions were significantly different from the control at the $3 \mathrm{rd}$ and 4 th generations $\left(\chi^{2}=\right.$ 42.17, $P<0.01$ in the 3rd generation, Figure 4c; and $\chi^{2}=158.33, P<0.01$ in the 4 th generation, Figure $4 d$ ). The proportion of larval survival in $R$ and $\mathrm{H}$ colonies in the 4 th generation showed an increase of $46.6 \%$ and $53.2 \%$, respectively, compared with that of the control $(P<0.01$ in both pairwise comparisons, Figure $4 d$ ). In particular, we found the copy number of SBV in SBV-killed larvae and survived pupae from SBV-inoculated colonies of breeding lines $(\mathrm{R}, \mathrm{H}$, and $\mathrm{R} \times \mathrm{H})$ in the fourth generation were significantly higher than those of the control (LSD post hoc tests, $P<0.01$ in all pairwise comparisons, Online resource $4 b$ ), indicating a higher tolerance of broods against SBV. Therefore, survival rates of larvae in $\mathrm{R} \times \mathrm{H}$ colonies reached $\sim 70 \%$ (Figure $4 \mathrm{~d}$ ), although this survivorship was not significantly different to those of SBV-uninfected colonies, which showed $82.5 \%$ survival rate $\left(\chi^{2}=4.73, \mathrm{R} \times \mathrm{H}\right.$ colonies vs. $\mathrm{SBV}$ free colonies, $P>0.05$, Figure $4 \mathrm{~d})$.

\subsection{Queen-destined brood survivorship}

The survival proportion of queen-destined broods, which were reared in SBV-infected nursery colonies, from one-day-old 1 st instar larvae to the emergence of virgin queens 

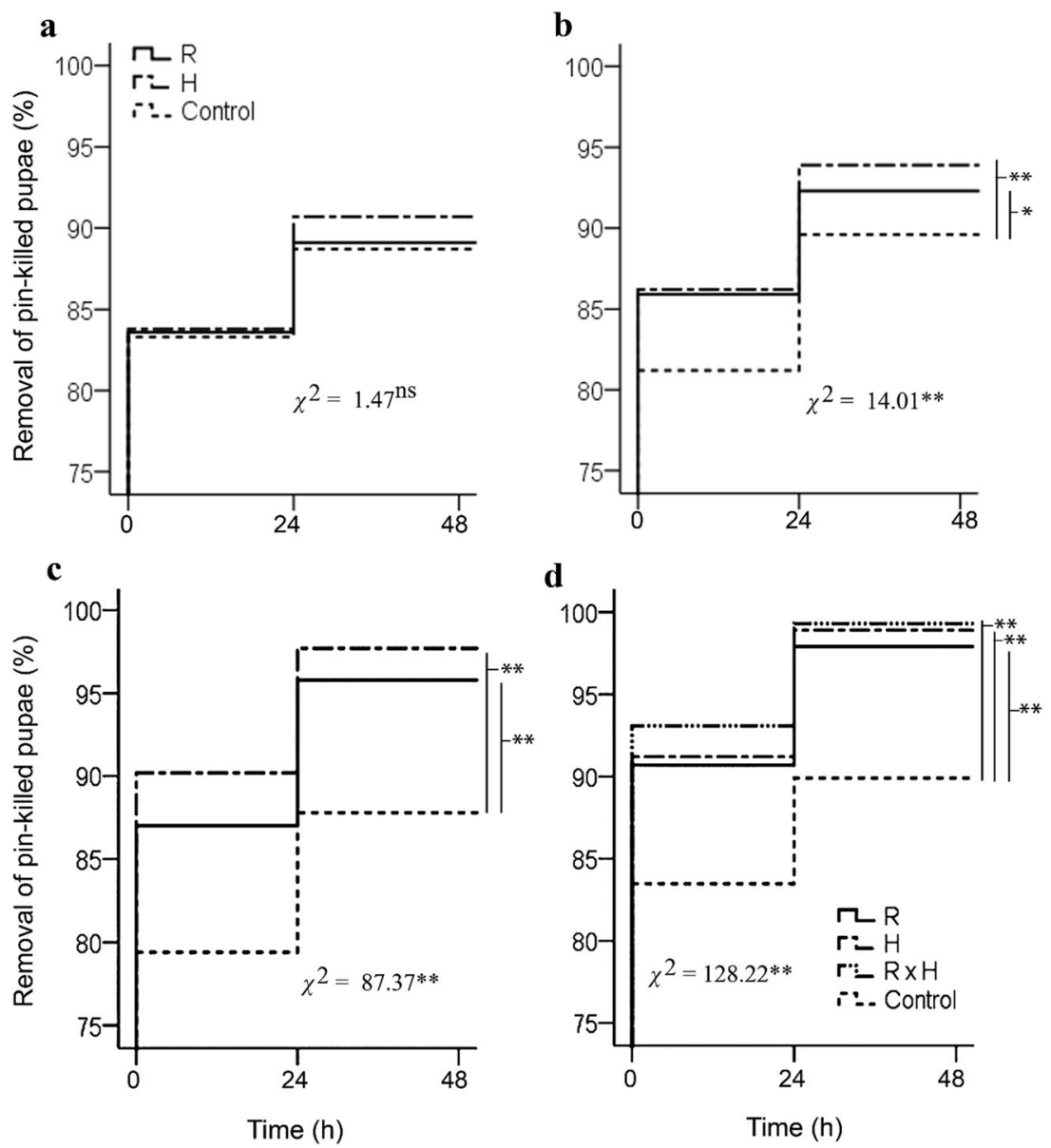

Figure 2. Proportion of pin-killed pupae removed over time from the colonies of breeding lines $(\mathrm{R}, \mathrm{H}$, and $\mathrm{R} \times \mathrm{H})$ and control in the first (a), second (b), third (c), and fourth (d) generations. $P$ values were obtained from the log-rank chi square $\left(\chi^{2}\right)$ tests, and log-rank post hoc (Mantel-Cox) tests are shown as follows: $* P<0.05 ; * * P<0.01$; and ns, no significant difference.

among experimental groups (lines $\mathrm{R}$ and $\mathrm{H}$ ) in the 1 st and 2 nd generation was not significantly different from that of the control $\left(\chi^{2}=0.02\right.$, $P=0.99$, Figure 5a; and $\chi^{2}=5.04, P=0.08$, Figure $5 b$ ). However, the survivorship of the experimental groups in the third generation was significantly different from that of the control $\left(\chi^{2}=26.61, P<0.01\right.$, Figure 5c). The survival proportions of queen-destined broods grafted from the colonies of lines $\mathrm{R}$ and $\mathrm{H}$ in the third generation were $30 \%$ and $32.5 \%$ higher than those of the controls, respectively $(P<0.01$ in both pairwise comparisons, Figure 5c).

\subsection{Number of pupae in SBV-inoculated colonies}

The number of sealed worker broods (pupae) in the colonies of breeding lines and control in all four generations were statically different (repeated measures ANOVA, $P<0.01$; Figure 6). Following SBV inoculation, the number of pupae in colonies of all groups in the 1st and 2nd generations gradually decreased over time. Consequently, the number of pupae in all colonies of these first two generations declined to a comparatively low value at 45 days post SBV inoculation (dpi), indicating a near entire colony collapse (Online 

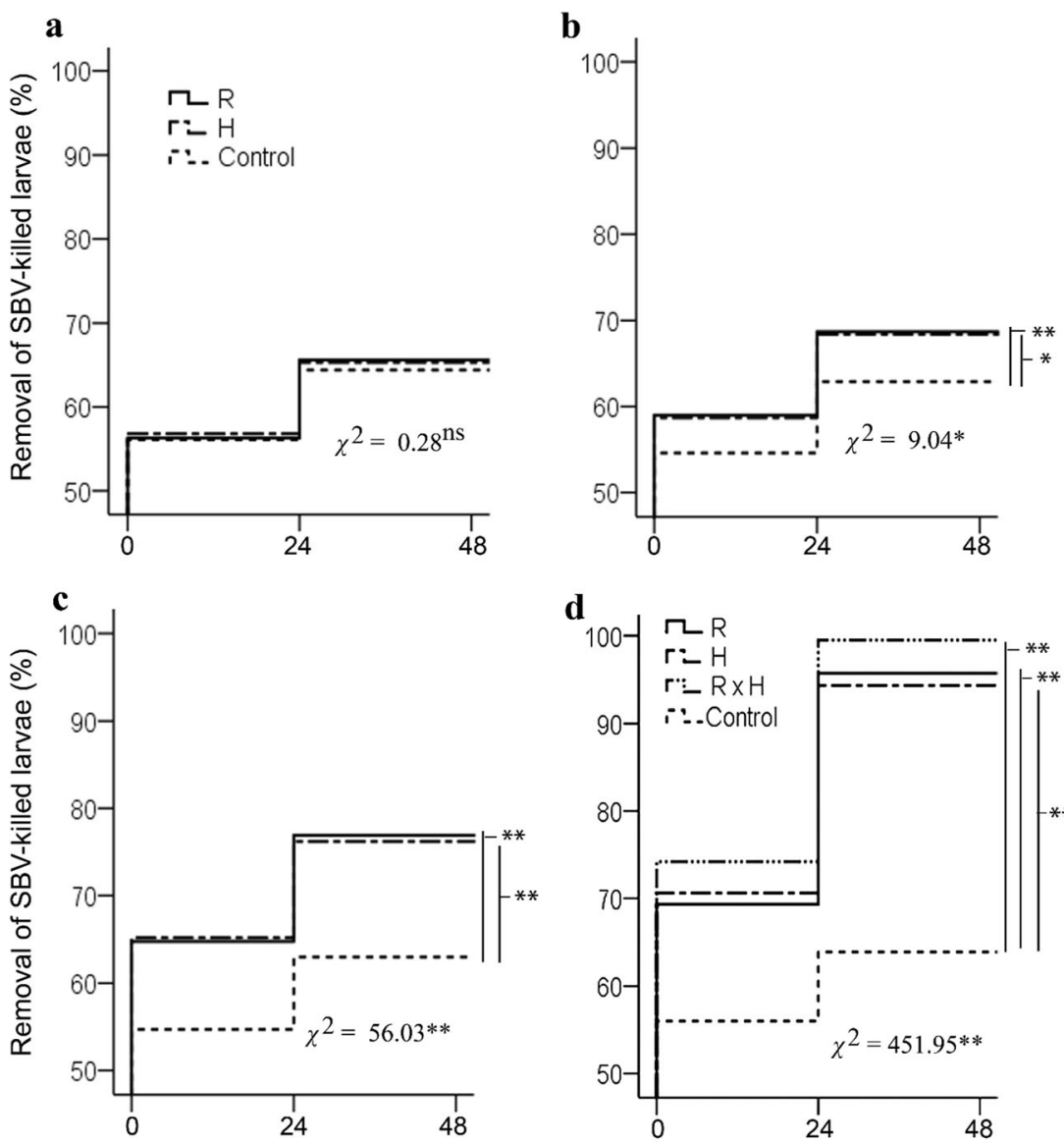

Time (h)

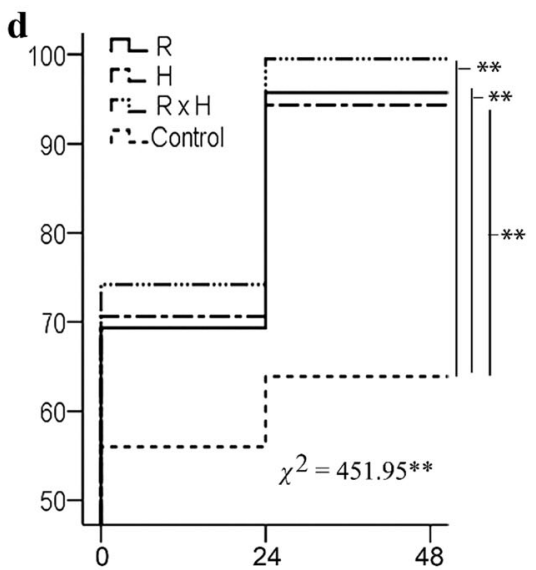

Time (h)

Figure 3. Proportion of SBV-killed larvae removed over time from the colonies of breeding lines $(\mathrm{R}, \mathrm{H}$, and $\mathrm{R} \times \mathrm{H})$ and the control group in the first (a), second (b), third (c), and fourth (d) generations. $P$ values were obtained from the log-rank chi square $\left(\chi^{2}\right)$ tests, and log-rank post hoc (Mantel-Cox) tests are shown as follows: $* P<0.05$; $* * P<$ 0.01 ; and ns, no significant difference.

resource 2). However, at $45 \mathrm{dpi}$, the number of pupae in colonies of breeding lines in all generations was significantly higher than those of the control $(P<0.01$ in all comparisons at $45 \mathrm{dpi}$; Figure 6). In the 3rd and 4th generations, the number of pupae in colonies of breeding lines was significantly different from the control $(P<$ 0.01 in all comparison; Figure $6 \mathrm{c}$ and d).

\section{DISCUSSION}

Our breeding program was aimed to breed honey bee lines with SBV resistance by selecting those with superior hygienic behavior and brood viability. This approach is consistent with previous breeding approaches, which have shown that honey bee colonies selected for hygienic behavior were also resistant to brood diseases (Gilliam et al. 1983; Palacio et al. 2000; Spivak and Reuter 2001). In this study, we confirmed the efficiency of the hygienic behavior against SBV disease, making it a crucial factor to be targeted for the selection of A. cerana bee lines. Steady increases in the removal proportion of pin-killed pupae and SBV-killed larvae throughout breeding generations (Figures 2 and 3 ) suggest that the hygienic mechanism is a heritable trait in A. cerana, an observation that was also found in Apis mellifera 

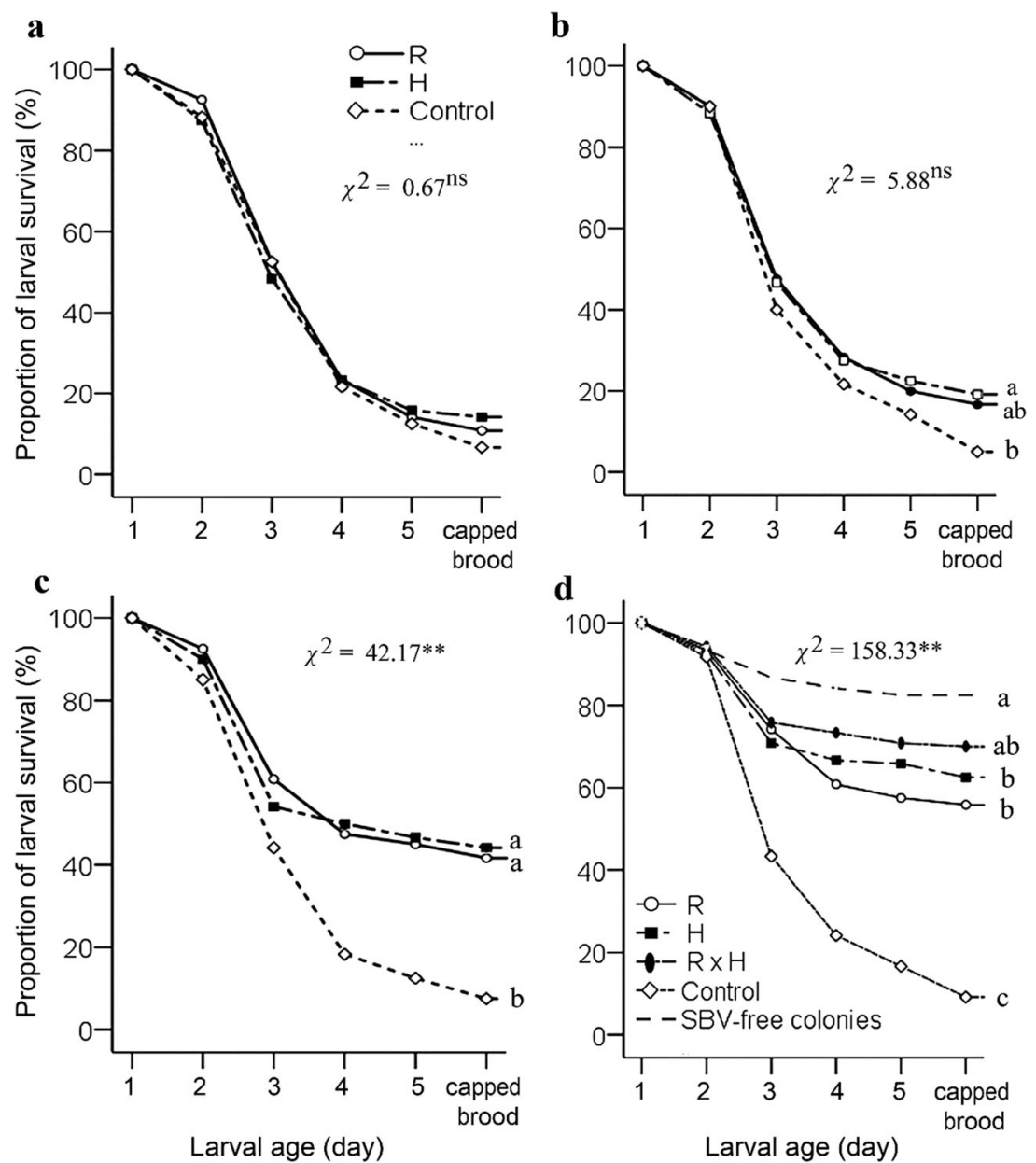

Figure 4. Proportion of larval survivorship in SBV-infected A. cerana colonies over larval stages in the first (a), second (b), third (c), and fourth (d) generations. $P$ values were obtained from the log-rank chi square $\left(\chi^{2}\right)$ test (ns, no significant difference at the $P<0.05$ level; *significant difference at the $P<0.05$ level; and $* *$ significant difference at the $P<0.01$ level). Different letters in the same graph indicate significant differences at the $P<0.05$ level (Mantel-Cox tests).

after intensive selection for hygienic behavior (Milne Jr 1985; Spivak and Reuter 2001; Lapidge et al. 2002). In addition, colony strength recovery 45 dpi (fourth generation, Figure $6 \mathrm{~d}$ and Online resource $2 \mathrm{~d}$ ) indicates that an improvement in SBV resistance is also feasible in A. cerana colonies by utilizing hygienic traits as selection criteria. This process was previously applied in A. mellifera breeding programs, showing that disease resistance of selected colonies was largely strengthened over several years of breeding programs (Gilliam et al. 1983; Palacio et al. 2000; Spivak and Reuter 2001).

Interestingly, we observed that A. cerana removed pin-killed pupae earlier and in a greater quantity than SBV-killed larvae. For instance, the removal proportion of pin-killed pupae in the first generation was about $84 \%$ and $90 \%$ at $24 \mathrm{~h}$ and 48 $\mathrm{h}$, respectively (Figure 2a), whereas that of SBVkilled larvae was about $56 \%$ and $65 \%$ at $24 \mathrm{~h}$ and $48 \mathrm{~h}$, respectively (Figure 3a). Obviously, the removal proportion of SBV-killed larvae at $48 \mathrm{~h}$ was 


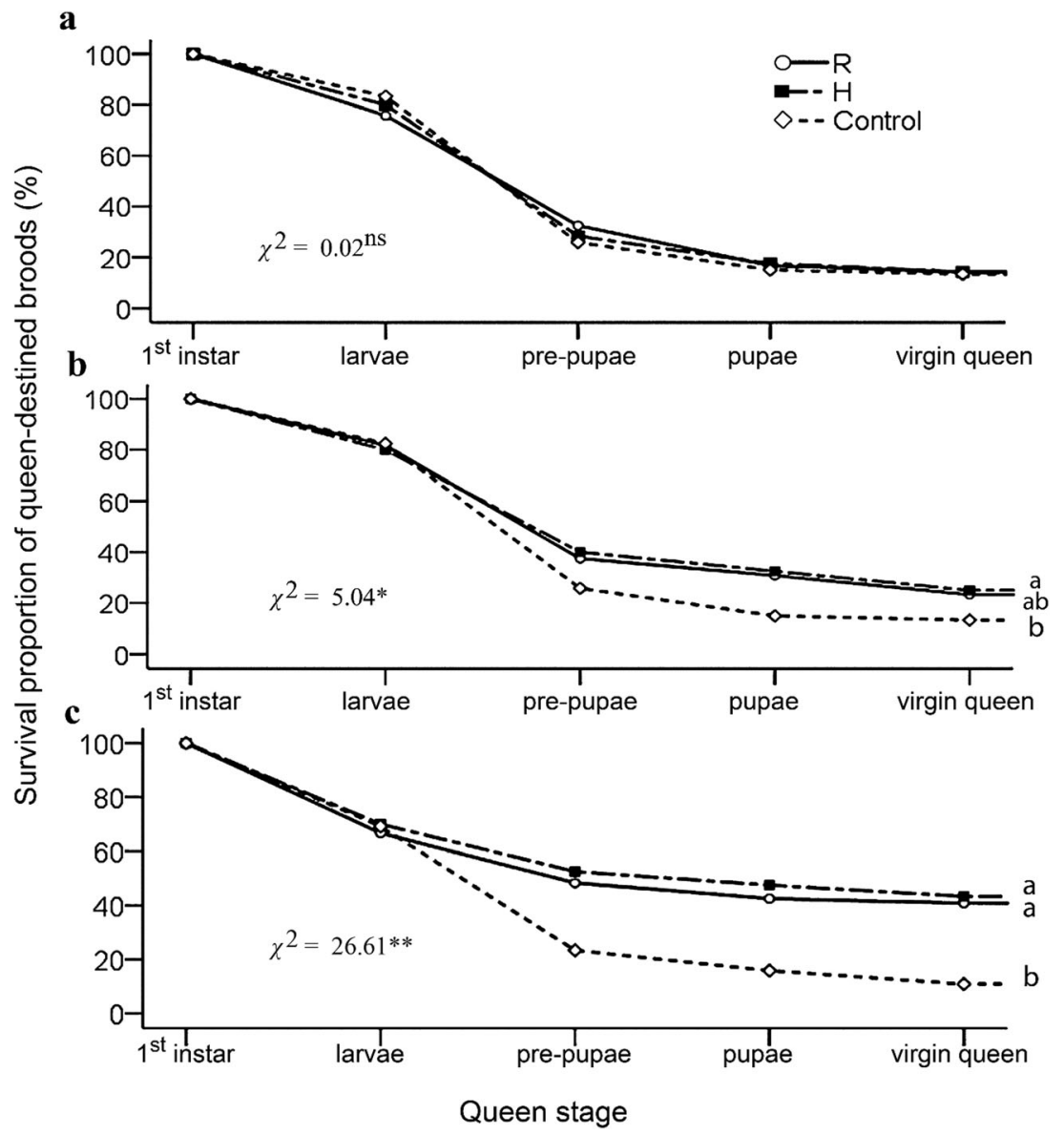

Figure 5. Proportion of A. cerana queen-destined brood survival over periods of different brood stages in the first (a), second (b), and third (c) generations. Survival rates of queen-destined broods at 6 and 11 days after larval graft to nursery colonies were recorded to calculate the ratio of larval survival and pupal survival, respectively. The remaining queen-destined broods in queen cells on the 11th day after larval graft to nursery colonies were considered dead after a visual examination of prepupal or pupal morphology. $P$ values were obtained from the log-rank chi square $\left(\chi^{2}\right)$ test (ns, no significant difference at the $P<0.05$ level; *significant difference at the $P<0.05$ level; and **significant difference at the $P<0.01$ level). Different letters in the same graph indicate significant differences at the $P<0.05$ level based on log-rank post hoc (Mantel-Cox) tests.

even lower than that of pin-killed pupae at $24 \mathrm{~h}$, indicating a less efficient hygienic behavior against SBV-infected broods in A. cerana. The delayed response of workers to SBV-infected larvae could be ascribed to the inability to remove the individuals infected with SBV disease due to poor sensitization of workers toward SBV-infected larvae, which consequently caused the severity of SBV disease in the infected colony. Previous studies have demonstrated that different olfactory cues emanating between diseased and killed broods were perceived differently by workers (Masterman et al. 2001; Palacio et al. 2010). This difference would be related to the different sensitivities of workers to the olfactory stimulus of diseased larvae and dead pupae that elicit subsequent hygienic behavior (Gramacho and Spivak 2003; Swanson et al. 2009; Palacio et al. 2010). Thus, these results may indicate the importance of careful inspection of actual removal activities of diseased broods by workers when $A$. cerana colonies are selected against specific pathogens. 

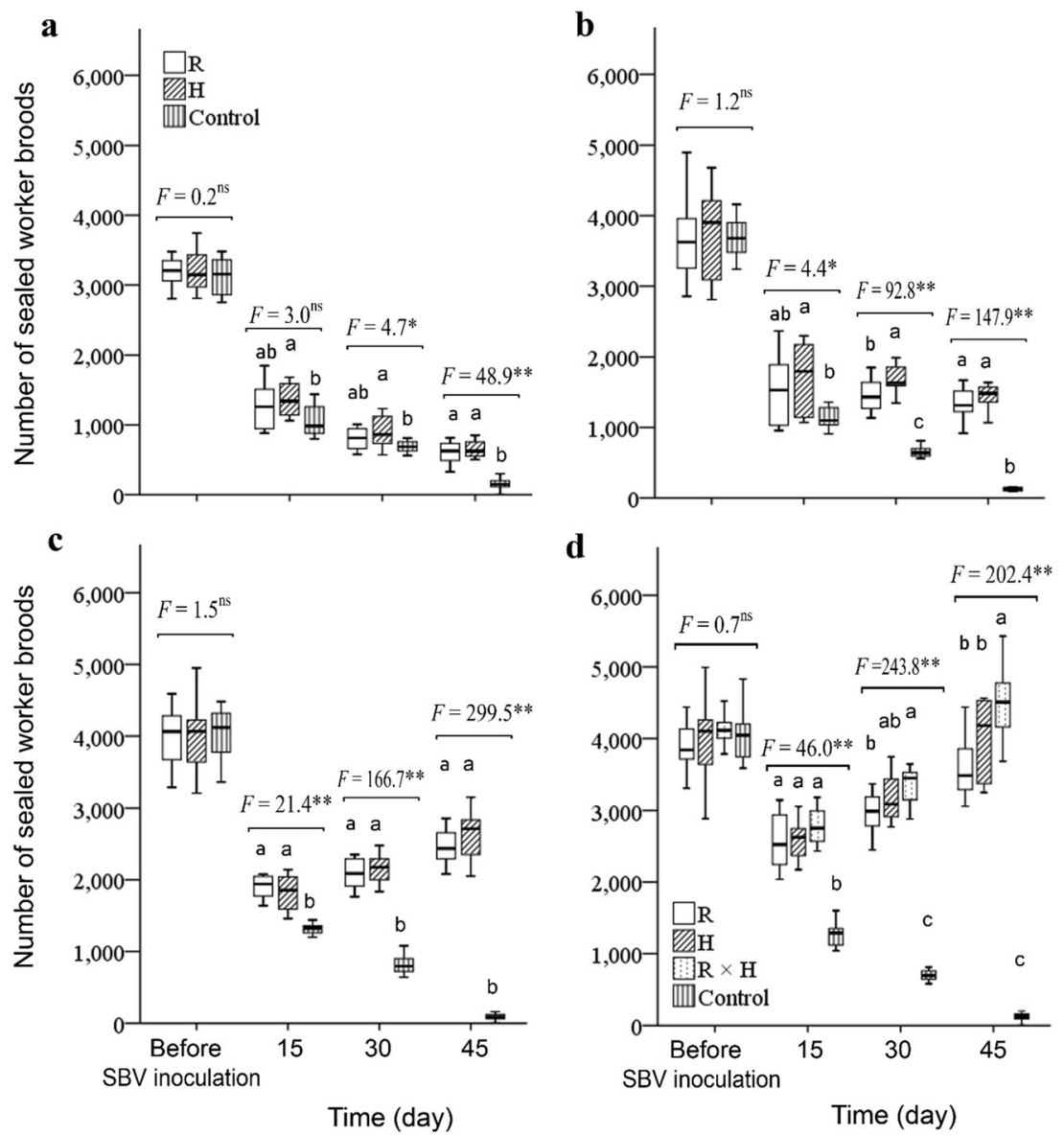

Time (day)

Figure 6. Number of sealed worker broods in SBV-inoculated colonies of breeding lines $(\mathrm{R}, \mathrm{H}$, and $\mathrm{R} \times \mathrm{H})$ and controls in the first (a), second (b), third (c), and fourth (d) generations. Boxplots demonstrate the quartiles (length of box) and median (horizontal line inside the box), and whiskers represent 1.5 times the interquartile range. $F$ value obtained from one-way ANOVA tests are shown as follows: $* P<0.05$; $* * P<0.01$; and ns, no significant difference. Different letters indicate significant differences at the $P<0.05$ level (Fisher's LSD tests).

Conceptually, early detection and rapid response of workers, which invoke the timely removal of infected broods, would be effective in reducing pathogen spread in colonies (Wilson-Rich et al. 2009). The initiation of hygienic performance was reported to be largely dependent on the stimulus response threshold of workers (Masterman et al. 2001). Gramacho and Spivak (2003) reported that workers in colonies selectively bred for highly hygienic behavior had a lower threshold for stimulus perception and greater sensitivity to olfactory cues associated with diseased broods than those of non-hygienic bees. Over four selective generations, colonies of our breeding lines also showed a faster hygienic removal and higher removal proportion of SBV-killed larvae than the control (Figure 3d). These improvements would be related to the abilities of hygienic workers, having better olfactory perception and response to the stimulus that elicits hygienic activities. Thus, workers with low stimulus thresholds for both the olfactory and behavior responses can rapidly perform hygienic removal of SBV-infected larvae from the colony. We believe that colonies of $A$. cerana selected for highly hygienic removal of SBV-killed larvae would provide an effective way to restrict SBV infection and 
transmission within colonies. Considering the increased survivorship of pupae and workers (Figure 6 and Online resource 2) and the brood survival ratio (Figures 4 and 5), the reduction in disease pressure positively influenced colony fitness.

Honey bees have a patriline system, which allows colonies to maintain genetic diversity and results in differentiated survivorship against diseases. In this study, we successfully raised several queen-destined larvae of the selected colonies in SBV-infected nursery colonies (Figure 5) as a screening for the selection of resistant individuals. The queen-destined broods that successfully survived the SBV-infected conditions and emerged as virgin queens could potentially have resistance against SBV. Subsequent offspring colonies obtained from these queens exhibited a gradual increase in brood survivorship throughout breeding generations (Figures 4 and 5). Indeed, previous study has demonstrated relatively higher levels of SBV infection in the larval stage than in the pupal and adult stages (Shan et al. 2017). Thus, it is conceivable that larvae that do not succumb to SBV infection and continue to grow to the adult stage are likely to have active immunity against SBV (Online resource 4b). This, therefore, explains that colonies of breeding lines $(\mathrm{R}, \mathrm{H}$, and $\mathrm{R} \times \mathrm{H}$ ) in the fourth generation were actually infected with SBV (Online resource 4), but the infected broods in those colonies could overcome the SBV infection during the larval and pupal stages, resulting in a higher proportion of brood survivorship (Figures 4d and 6d). An increase in brood survivorship under SBV-infected conditions suggests that SBV-tolerant capabilities at the individual level could be improved over breeding generations. Our results are in line with previous findings that selection for resistant honey bee against viral disease is feasible over several generations (Kulinčević and Rothenbuhler 1975)

Genetic diversity also has an effect on colony growth and subsequent honey production. We found a significantly higher number of workers in $\mathrm{R} \times \mathrm{H}$ colonies under SBV-uninfected conditions at 60 and 80 days after colony establishment than in controls (Online resource 3a). This higher number of workers consequently resulted in higher honey production (Online resource $3 \mathrm{~b}$ ). In addition, our results indicated that the number of pupae and workers in $\mathrm{R} \times \mathrm{H}$ colonies 45 dpi not only were significantly differed from those of the control but also were higher than the mean values of their parental colonies ( $\mathrm{R}$ and $\mathrm{H}$; Figure $6 \mathrm{~d}$ and Online resource $2 \mathrm{~d}$ ). This suggests that genetic variability that stemmed from the crossbreeding of two separate lines (i.e., $\mathrm{R}$ and $\mathrm{H}$ ) may have had an influence on the colony's fitness and SBV-resistance. In honey bee breeding, there is a phenomenon of genetic drift resulting in an inbreeding depression due to the consequence of bottlenecking event. Thus, colonies headed by an inbred queen suffer from low brood viability due to the increase of diploid drones (Zayed 2004). Previous studies have shown that genetic diversity at the colony level promoted disease resistance and colony fitness (Tarpy 2003; Mattila and Seeley 2007), indicating the importance of maintaining high genetic diversity among breeding lines. Our approach is in line with previous breeding programs, which maintained separate breeding lines for subsequent crossbreeding to produce hybrid queens for the improvement of genetic traits without sacrificing genetic diversity (Moritz 1984; Chapman et al. 2008).

In summary, we bred SBV-resistant A. cerana from the selection of colonies exhibiting superior hygienic behavior and brood viability. Our results emphasize that the strategy to select colonies with high hygienic behavior and brood viability is practical.

\section{AUTHORS CONTRIBUTION}

NNV and YSC conceived the study, performed experiments, and analyzed data; NNV wrote the draft and participated in revisions; YSC was involved in writing an early version of the paper; IK schemed and organized the data and revised and finalized the paper. All the authors read and approved the final version of the manuscript.Funding information

This study was supported by a research project (grant number PJ014180022019) from the National Institute of Agricultural Sciences, Rural Development Administration, Republic of Korea. 


\section{COMPLIANCE WITH ETHICAL STANDARDS}

Conflict of interest The authors declare that they have no conflict of interest.

Haute résistance à la maladie du couvain sacciforme dans les colonies d' Apis cerana (Hymenoptera: Apidae) sélectionnées pour la viabilité supérieure du couvain et leur comportement hygiénique.

Apis cerana / apiculture / Sacbrood bee virus (SBV) / insémination instrumentale / maladie du couvain.

Hohe Resistenz gegenüber Sackbrutvirus-Infektionen in Völkern von Apis cerana (Hymenoptera: Apidae), die auf bessere Brut-Viabilität und Hygieneverhalten selektiert wurden.

Apis cerana / bienenzucht / Sackbrutvirus / instrumentelle besamung / brutkrankheiten.

\section{REFERENCES}

Bailey, L., Gibbs, A.J., Woods, R.D. (1964) Sacbrood virus of the larval honey bee (Apis mellifera Linnaeus). Virology 23, 425-429.

Baker, A.C., Schroeder, D.C. (2008) The use of RNAdependent RNA polymerase for the taxonomic assignment of Picorna-like viruses (order Picornavirales) infecting Apis mellifera L. populations. Virol. J. 5, 10.

Brutscher, L.M., Daughenbaugh, K.F., Flenniken, M.L. (2015) Antiviral defense mechanisms in honey bees. Curr. Opin. Insect Sci. 10, 71-82.

Chapman, N.C., Lim, J., Oldroyd, B.P. (2008) Population genetics of commercial and feral honey bees in Western Australia. J. Econ. Entomol. 101 , 272-277.

Chen, Y.P., Siede, R. (2007) Honey bee viruses, Adv. Virus Res., Elsevier Academic Press Inc., San Diego, pp. 3380 .

Choe, S.E., Nguyen, L.T.K., Noh, J.H., Koh, H.B., Jean, Y.H., Kweon, C.H., Kang, S.W. (2012) Prevalence and distribution of six bee viruses in Korean Apis cerana populations. J. Invertebr. Pathol. 109, 330-333.

Choi, Y.S., Lee, M.Y., Hong, I.P., Kim, N.S., Kim, H.K., Lee, K.G., Lee, M.L. (2010) Occurrence of Sacbrood virus in Korean apiaries from Apis cerana (Hymenoptera: Apidae). Korean J. Apic. 25, 187-191.

Cobey, S.W., Tarpy, D.R., Woyke, J. (2013) Standard methods for instrumental insemination of Apis mellifera queens. J. Apic. Res. 52, 1-18.

Corlett, R.T. (2004) Flower visitors and pollination in the Oriental (Indomalayan) region. Biol. Rev. Camb. Philos. Soc. 79, 497-532.

Cremer, S., Sixt, M. (2009) Analogies in the evolution of individual and social immunity. Philos. Trans. R. Soc. B 364, 129-142.

Cremer, S., Pull, C.D., Fürst, M.A. (2018) Social immunity: Emergence and evolution of colony-level disease protection. Annu. Rev. Entomol. 63, 105-123.

DeFelice, D., Ross, C., Simone-Finstrom, M., Warrit, N., Smith, D.R., Burgett, M., Sukumalanand, P., Rueppell, O. (2015) Geographic variation in polyandry of the Eastern Honey Bee, Apis cerana, in Thailand. Insect. Soc. 62, 37-42.

Evans, J.D., Spivak, M. (2010) Socialized medicine: individual and communal disease barriers in honey bees. J. Invertebr. Pathol. 103, 62-72.

Evans, J.D., Aronstein, K., Chen, Y.P., Hetru, C., Imler, J.L., Jiang, H., Hultmark, D. (2006) Immune pathways and defence mechanisms in honey bees Apis mellifera . Insect Mol. Biol. 15, 645-656.

Gilliam, M., Tarber, S., Richardson, G.V. (1983) Hygienic behavior of honey bees in relation to Chalkbrood disease. Apidologie 14, 29-39.

Gong, H.R., Chen, X.X., Chen, Y.P., Hu, F.L., Zhang, J.L., Lin, Z.G., Yu, J.W., Zheng, H.Q. (2016) Evidence of Apis cerana Sacbrood virus infection in Apis mellifera. Appl. Environ. Microbiol. 82, 2256-2262.

Gramacho, K.P., Spivak, M. (2003) Differences in olfactory sensitivity and behavioral responses among honey bees bred for hygienic behavior. Behav. Ecol. Sociobiol. 54, 472-479.

Honey Bee Genome Sequencing Consortium (2006) Insights into social insects from the genome of the honeybee Apis mellifera. Nature 443, 931-949.

Kulinčević, J.M., Rothenbuhler, W.C. (1975) Selection for resistance and susceptibility to hairless-black syndrome in the honeybee. J. Invertebr. Pathol. 25, 289295.

Lapidge, K.L., Oldroyd, B.P., Spivak, M. (2002) Seven suggestive quantitative trait loci influence hygienic behavior of honey bees. Naturwissenschaften $\mathbf{8 9}$, 565-568.

Lin, Z., Page, P., Li, L., Qin, Y., Zhang, Y., Hu, F., Neumann, P., Zheng, H., Dietemann, V. (2016) Go east for better honey bee health: Apis cerana is faster at hygienic behavior than A. mellifera. PLoS One 11, e0162647.

Masterman, R., Ross, R., Mesce, K., Spivak, M. (2001) Olfactory and behavioral response thresholds to odors of diseased brood differ between hygienic and nonhygienic honey bees (Apis mellifera L.). J. Comp. Physiol. A. 187, 441-452. 
Mattila, H.R., Seeley, T.D. (2007) Genetic diversity in honey bee colonies enhances productivity and fitness. Science 317, 362-364.

McMenamin, A.J., Daughenbaugh, K.F., Parekh, F., Pizzorno, M.C., Flenniken, M.L. (2018) Honey bee and bumble bee antiviral defense. Viruses 10, 395.

Milne Jr, C.P. (1985) Estimates of the heritabilities of and genetic correlation between two components of honey bee (Hymenoptera: Apidae) hygienic behavior: uncapping and removing. Ann. Entomol. Soc. Am. 78, 841-844.

Moritz, R.F.A. (1984) Selection in small populations of the honeybee (Apis mellifera L.). J. Anim. Breed. Genet. $\mathbf{1 0 1}, 394-400$.

Newton, D.C., Ostasiewski, N.J. (1986) A simplified bioassay for behavioral resistance to American foulbrood in honey bees (Apis mellifera L). Am. Bee J. 126, 278-281.

Oldroyd, B.P., Clifton, M.J., Parker, K., Wongsiri, S., Rinderer, T.E., Crozier, R.H. (1998) Evolution of mating behavior in the genus Apis and an estimate of mating frequency in Apis cerana (Hymenoptera: Apidae). Ann. Entomol. Soc. Am. 91, 700-709.

Palacio, M.A., Figini, E.E., Ruffinengo, S.R., Rodriguez, E.M., del Hoyo, M.L., Bedascarrasbure, E.L. (2000) Changes in a population of Apis mellifera L. selected for hygienic behaviour and its relation to brood disease tolerance. Apidologie 31, 471-478.

Palacio, M.A., Rodriguez, E., Goncalves, L., Bedascarrasbure, E., Spivak, M. (2010) Hygienic behaviors of honey bees in response to brood experimentally pin-killed or infected with Ascosphaera apis. Apidologie 41, 602-612.

Rath, W. (1999) Co-adaptation of Apis cerana Fabr. and Varroa jacobsoni Oud. Apidologie 30, 97-110.

Rosenkranz, P., Tewarson, N.C., Singh, A., Engels, W. (1993) Differential hygienic behaviour towards Varroa jacobsoni in capped worker brood of Apis cerana depends on alien scent adhering to the mites. J. Apic. Res. 32, 89-93.
Shan, L., Liuhao, W., Jun, G., Yujie, T., Yanping, C., Jie, W., Jilian, L. (2017) Chinese Sacbrood virus infection in Asian honey bees (Apis cerana cerana) and host immune responses to the virus infection. J. Invertebr. Pathol. 150, 63-69.

Siva-Jothy, M.T., Moret, Y., Rolff, J. (2005) Insect immunity: an evolutionary ecology perspective. Adv. Insect Phys. 32, 1-48.

Spivak, M., Reuter, G.S. (2001) Resistance to American foulbrood disease by honey bee colonies Apis mellifera bred for hygienic behavior. Apidologie 32, 555-565.

Swanson, J.A., Torto, B., Kells, S.A., Mesce, K.A., Tumlinson, J.H., Spivak, M. (2009) Odorants that induce hygienic behavior in honeybees: identification of volatile compounds in Chalkbrood-infected honeybee larvae. J. Chem. Ecol. 35, 1108-1116.

Tarpy, D.R. (2003) Genetic diversity within honeybee colonies prevents severe infections and promotes colony growth. Proc. Biol. Sci. 270, 99-103.

Theisen-Jones, H., Bienefeld, K. (2016) The Asian honey bee (Apis cerana) is significantly in decline. Bee World 93, 90-97.

Wilson-Rich, N., Spivak, M., Fefferman, N.H., Starks, P.T. (2009) Genetic, individual, and group facilitation of disease resistance in insect societies. Annu. Rev. Entomol. 54, 405-423.

Wu, Y., Dong, X., Kadowaki, T. (2017) Characterization of the copy number and variants of Deformed Wing Virus (DWV) in the pairs of honey bee pupa and infesting varroa destructor or Tropilaelaps mercedesae. Front. Microbiol. 8, 1558.

Zayed, A. (2004) Effective population size in Hymenoptera with a complementary sex determination. Heredity $\mathbf{9 3}$, 627-630.

Publisher's note Springer Nature remains neutral with regard to jurisdictional claims in published maps and institutional affiliations. 